\title{
A Bayes Random Fields Approach for Integrative Large-Scale Regulatory Network Analysis
}

\author{
Yinyin Yuan ${ }^{1,2}$ and Chang-Tsun $\mathrm{Li}^{1}$ \\ ${ }^{1}$ Department of Computer Science, University of Warwick, Coventry, UK
}

\begin{abstract}
Summary
We present a Bayes-Random Fields framework which is capable of integrating unlimited data sources for discovering relevant network architecture of large-scale networks. The random field potential function is designed to impose a cluster constraint, teamed with a full Bayesian approach for incorporating heterogenous data sets. The probabilistic nature of our framework facilitates robust analysis in order to minimize the influence of noise inherent in the data on the inferred structure in a seamless and coherent manner. This is later proved in its applications to both large-scale synthetic data sets and Saccharomyces Cerevisiae data sets. The analytical and experimental results reveal the varied characteristic of different types of data and refelct their discriminative ability in terms of identifying direct gene interactions.
\end{abstract}

\section{Introduction}

The most significant recent advances in system biology have been the developments of highthroughput techniques to produce large-scale genomic, transcriptomic and proteomic data. Meanwhile, huge amount of data incurs difficulties in information exploitation. This entails objective techniques to summarize findings from these data.

On the other hand, one major aim in system biology is the reverse engineering of transcriptional regulatory network, which brings the understanding of organisms at a genomic level. In the hope of discovering transcriptional regulatory activities, one promising research direction is the integration of diverse data types. For example, microarray gene expression data and transcription factor binding data can indicate the functional network in different aspects and on different levels, based on their different features. Whereas gene expression data often lack the degree of specificity, binding data are generally more specific but also significantly more sparse. Therefore, by integrating multiple data types, one can expect false positives to be reduced and disparities between different levels of the system to be identified. Further, integration helps to explain complex biological interaction on a deeper level than using a single data alone [1].

A key challenge in data integration is the development of a robust system that can be routinely applied to heterogeneous and noisy data. However, such system has not yet been proposed. The reasons are manifold. First biological data are quite of different nature and formats. For example, gene expression data are often multi-dimensional if they are sampled over time, whilst most of the other data types are one-dimensional. The problem becomes how to facilitate effective integration on data of different sizes. Another reason is that the coverage of each data type is different from each other. For instance, while gene expression data cover almost

\footnotetext{
${ }^{2}$ To whom correspondence should be addressed. E-mail: yina@dcs .warwick. ac.uk
} 
the entire genome, transcription factor binding data can only partially cover the interactions between transcription factors and other genes. The system has therefore to be carefully designed to retain elegance and flexibility.

We propose a Bayes-Random Fields approach (BRFs) for integrative analysis of large-scale regulatory network. It is our hope that such analysis reveals the elementary structure of regulatory interactions responsible for higher level properties of organisms such as cell growth and death. Our inspiration comes from the similarity between the transcriptional network reverse engineering problem and the inverse problem in signal processing [2]. Over the past decades, robust statistical methods have matured into some of the most powerful techniques to extract meaningful conclusions from a diversity of data types. The context is similar to the newly arisen study of biological data integration. However, instead of rigidly relying on existing techniques, we take into account diverse features of biological data for full utilization.

Very often, integrative systems are constrained to certain two or three types of data $[1,3,4]$. For example, protein-protein interaction data and gene expression data are used in constructing probabilistic network models [3]. Sun et al. [5] integrates transcription factor binding data and gene expression data by treating transcription activity represented by expression as a result of transcription factor binding. Another research gap is that usually only a small number of genes can be incorporated into a network due to the inefficiency of learning techniques. However, it is necessary to put the regulatory relationships in a larger context, both because transcriptional activities are usually multi-stages and operate like chain actions involving a large number of genes, and that gene regulations are typically embedded in a vast network of biochemistry interactions [6].

As the first attempt towards general integrative analysis, a Bayesian framework [7] has been proposed based on similarity matrices inferred from heterogeneous types of biological data. Later, Rhodes et al. [8] applied a Naïve Bayesian Network model which defines a distribution as a product of disjoint classes, in this case, dataset-specific likelihood ratios. However, its multiplicative nature requires that the attributes are conditionally independent given the classes, which remains debatable. Our proposed framework differs from theirs by using a stochastic process so as to exclude the noise presented in most of the data, while remains efficient enough to facilitate large-scale analysis.

Since the focus of this study is the structure of large-scale networks, we only consider undirected graphs. Previous studies have shown that the nature of a network can be recovered even if it is undirected [9]. Moreover, an undirected graph is conceptually simple in the sense that the problem with feedback loops as in a Bayesian network is out of the question, hence is more widely applicable, especially in integrative study when some of the data may be undirectional (for example gene ontology categories [10]).

After performing extensive evaluations on microarray data (described in Section 3 in the supplement file), we propose a full Bayesian approach to incorporate not only microarray time series data, but also other heterogenous data types into a integrative regulatory network. Further, evaluations on the proposed framework are conducted on synthetic data sets of different sizes to show performance improvements. Finally, real Yeast data are integrated to construct a condition-specific network. The result from the proposed method is suggestive of a scale-free network with long tail distribution of the degree of connectivity. 


\section{Bayes Random Fields (BRFs) Integration}

In integrative study, microarray time series data attract special attention, not only because of their informative dynamic features, but also for the difficulty involved in the analysis due to their high dimensionality. In Section 3 in the supplement file, we evaluate two widely used methods for inference from gene expression data and as a result choose partial correlation of gene expression data for the proposed framework. Partial correlation (PCOR) is indicative of direct interactions between a pair of variables/genes by eliminating the effects from the rest of variables/genes and therefore has been popular in the research towards the inference of gene regulatory relationship [11]. In other words, if the residuals of two time series, after removing the effect from the rest of the data by regression, are still correlated, there exists direct interaction between them. A detailed description of PCOR and an efficient way of its computation are provided in Section 2 in the supplement file.

Since gene expression PCOR is used in our framework, the inputs of the system can be unified into probability matrices. Each entry in the matrices can indicate the probability of interaction between a pair of genes, that is, the probability that an edge exists between them in the network. Let $G$ denote the set of $n$ genes in the network and $X$ denote the edges, now the problem can be formulated as inferring a graphical interaction model $M=(G, X)$ from $m$ data sets such as gene expression PCOR and transcription factor binding data, each represented as a matrix of dimension $n \times n$. Let $p(X)$ denote a probability density over hidden variables/edges $X=\left\{x_{l} \mid l=1,2, \ldots, e\right\}$ with $e$ the total number of edges, $e=n(n-1) / 2$. Now we define a Bayes framework for integrative analysis with a Random Fields model as described in Section 2.1.

\subsection{Bayes framework}

The problem becomes how to integrate the information of the $m$ data matrices $\left\{\psi_{i} \mid i=1,2, \ldots, m\right\}$ and how to extract regulatory relationships summarized by a common feature $X$ in these data. Suppose each data matrix represents a property of $X,\left\{\phi_{i} \mid i=1,2, \ldots, m\right\}$, with Gaussian noise $\left\{\varepsilon_{i} \mid i=1,2, \ldots, m\right\}$, then we have

$$
\psi_{i}=\phi_{i}+\varepsilon_{i}, \quad i=1,2, \ldots, m
$$

Now we can set up a model using $X$ as the common feature/hidden variables among all the data. The objective is to estimate directly from $\psi$ not only $\phi$ but also their common feature $X$. The problem can be formulated as:

$$
\begin{aligned}
p\left(\phi_{1}, \ldots, \phi_{m}, X \mid \psi_{1}, \ldots, \psi_{m}\right)= & p\left(\phi_{1}, \ldots, \phi_{m} \mid X, \psi_{1}, \ldots, \psi_{m}\right) p\left(X \mid \psi_{1}, \ldots, \psi_{m}\right) \\
\propto & p\left(\psi_{1} \mid \phi_{1}, X\right) \cdots p\left(\psi_{m} \mid \phi_{m}, X\right) p\left(\phi_{1} \mid X\right) \\
& \cdots p\left(\phi_{m} \mid X\right) p\left(\psi_{1} \mid X\right) \cdots p\left(\psi_{m} \mid X\right) p(X) \\
\propto & p(X) \prod_{i=1}^{m} p\left(\psi_{i} \mid \phi_{i}\right) p\left(\phi_{i} \mid X\right) p\left(\psi_{i} \mid X\right)
\end{aligned}
$$

Thus in order to get $p\left(\phi_{1}, \ldots, \phi_{m}, X \mid \psi_{1}, \ldots, \psi_{m}\right)$, we need to define $p\left(\psi_{i} \mid \phi_{i}\right), p\left(\phi_{i} \mid X\right), p\left(\psi_{i} \mid X\right)$ and finally $p(X)$. The definitions of the first three probabilities are straightforward. Suppose $\varepsilon_{i}$ is Gaussian with mean equal to 0, according to Eq. (1) we have

$$
p\left(\psi_{i} \mid \phi_{i}\right)=\mathcal{N}\left(\phi_{i}, \sigma_{\varepsilon_{i}}^{2}\right)=\frac{1}{\left(\sqrt{2 \pi} \sigma_{\varepsilon_{i}}\right)^{e}} \exp \left\{-\frac{\left(\psi_{i}-\phi_{i}\right)^{2}}{2 \sigma_{\varepsilon_{i}}^{2}}\right\}
$$


The hidden variables $X$ has two classes, 0 and 1, representing the non-existence and existence of an edge respectively. We can assume that the probability density function in the two classes can be characterized by $N\left(\mu_{i 0}, \sigma_{i 0}^{2}\right)$ and $\mathcal{N}\left(\mu_{i 1}, \sigma_{i 1}^{2}\right)$,

$$
p\left(\phi_{i} \mid X\right)=\frac{1}{\left(\sqrt{2 \pi} \sigma_{i 0}\right)^{e_{0}}} \exp \left\{-\frac{\left(\phi_{i}-\mu_{i 0}\right)^{2}}{2 \sigma_{i 0}^{2}}\right\} \cdot \frac{1}{\left(\sqrt{2 \pi} \sigma_{i 1}\right)^{e_{1}}} \exp \left\{-\frac{\left(\phi_{i}-\mu_{i 1}\right)^{2}}{2 \sigma_{i 1}^{2}}\right\}
$$

where $e_{0}$ and $e_{1}$ denote the number of edges in state 0 and state $1, e_{0}+e_{1}=e$. With the same principle, we assign $p\left(\psi_{i} \mid X\right)$ by using Eq. (1), Eq. (4) and $p\left(\varepsilon_{i}\right)$ to the following

$$
\begin{aligned}
p\left(\psi_{i} \mid X\right)= & \mathcal{N}\left(\mu_{i 0}, \sigma_{i 0}^{2}+\sigma_{\varepsilon_{i}}^{2}\right) \cdot \mathcal{N}\left(\mu_{i 1}, \sigma_{i 1}^{2}+\sigma_{\varepsilon_{i}}^{2}\right) \\
= & \frac{1}{\left(\sqrt{2 \pi\left(\sigma_{i 0}^{2}+\sigma_{\varepsilon_{i}}^{2}\right)}\right)^{e_{0}}} \exp \left\{-\frac{\left(\psi_{i}-\mu_{i 0}\right)^{2}}{2\left(\sigma_{i 0}^{2}+\sigma_{\varepsilon_{i}}^{2}\right)}\right\} \\
& \cdot \frac{1}{\left(\sqrt{2 \pi\left(\sigma_{i 1}^{2}+\sigma_{\varepsilon_{i}}^{2}\right)}\right)^{e_{1}}} \exp \left\{-\frac{\left(\psi_{i}-\mu_{i 1}\right)^{2}}{2\left(\sigma_{i 1}^{2}+\sigma_{\varepsilon_{i}}^{2}\right)}\right\}
\end{aligned}
$$

$p(X)$ is defined in a random fields model below.

\subsection{Random Fields Model}

To estimate $p(X)$, a random fields model is desirable to represent a known feature of the gene network. A widely accepted concept in transcriptomics is the co-regulation within a gene cluster (co-expression), which can be interpreted as that if a gene is regulating most of the genes in a cluster, it is likely that links also exist between this gene and other genes in the same cluster. In the context of gene network modelling, we define the following model to represent this feature.

To define our clusters, we first perform cluster assignments for genes. The genes are clustered into $z$ clusters $\left\{C_{i} \mid i=1,2, \ldots, z\right\}$ using gene expression data, preferably by a tight clustering algorithm [12] designed for gene expression time series data. The purpose of applying this method is to obtain relatively small/tight clusters, so that relevant information based on these clusters can be inferred by the random fields model. This clustering method is also unsupervised in a sense that number of clusters needs not be specified.

The potential function in the random fields model is defined on the edges, i.e., between pairs of genes. Let $h_{i, C_{j}}$ be the number of edges between gene $i$ and cluster $j, d_{i}$ is the degrees of connectivity for gene $i$. The random fields method is formulated as the sum of potentials on all possible edges:

$$
p(X) \propto \exp \left[\sum_{i} \sum_{j \neq i} \omega_{i j}\left(h_{i, C_{j}}+h_{j, C_{i}}-\left(d_{i} / z\right)^{2}-\left(d_{j} / z\right)^{2}\right)\right]
$$

where $\omega_{i j}=\left(\left|C_{i}\right| \cdot\left|C_{j}\right|\right)^{-1}$ is a normalizing factor. The first two terms in the cost function represents the number of edges between gene $i$ and the cluster which gene $j$ belongs to, and vice versa, while the last two terms are the expected number of edges connecting gene $i$ and cluster $C_{j}$, and vice versa. The rationale supporting the potential function is that since co-expression 
indicates co-regulation, a higher potential should be given to the interaction between a pair of genes, if the existing interactions between their clusters are more than expected. An advantage of introducing such dependency is that it imposes a cluster constrain as a known gene network feature and iteratively refines the territory currently under evaluation.

\subsection{Gibbs sampling algorithm}

Let $\theta$ denote the parameter set $\left\{\theta_{i} \mid i=1,2, \ldots, m\right\}, \theta_{i}=\left\{\mu_{i 0}, \mu_{i 1}, \sigma_{i 0}, \sigma_{i 1}, \sigma_{\varepsilon_{i}}\right\}$. Jointly sampling the whole set $\left\{\phi_{i}, \theta_{i}, X\right\}$ from large-scale data $\psi_{i}$ is intractable. Since now all the variables of interest can be estimated by conditioning on the others, Gibbs sampling can be used to cycle through these conditional statements. By iteratively conditioning on the interim values of all other variables, Gibbs sampler aims to empirically approximate the desired marginal distribution for each variable. We assign a posteriori law

$$
\begin{aligned}
p(\phi, \theta, X \mid \psi) & =p(\phi, X \mid \theta, \psi) p(\theta \mid \phi, X, \psi) \\
& =p(\phi \mid X, \theta, \psi) p(X \mid \psi, \theta) p(\theta \mid \phi, X, \psi) \\
& =\prod_{i}^{m} p\left(\phi_{i} \mid X, \theta_{i}, \psi_{i}\right) p\left(X \mid \psi_{i}, \theta_{i}\right) p\left(\theta_{i} \mid \phi_{i}, X, \psi_{i}\right)
\end{aligned}
$$

Thus given data $\left\{\psi_{i} \mid i=1,2, \ldots, m\right\}$,

the Gibbs sampling algorithm is formulated as the following:

1. Initialization:

(a) First a random initial value $X^{(0)}$ is assigned.

(b) The conjugate priors for the hyperparameter variance $\sigma_{i k}(k \in\{0,1\})$ and $\sigma_{\varepsilon_{i}}$ in the normal distribution model are the inverse gamma distributions $(\mathcal{I G})$ [13], while for the hyperparameter mean $\mu_{i}$ it is given a normal prior. Therefore, first the hyperhyperparameters $\alpha_{i}, \beta_{i}, v_{i}, s_{i}^{2}, \alpha_{\varepsilon_{i}}, \beta_{\varepsilon_{i}}, i \in\{1,2, \ldots, m\}$ are assigned. Then the priors are sampled from the following distributions:

$$
\begin{aligned}
\sigma_{i k}^{2} & \sim \mathcal{I} \mathcal{G}\left(\alpha_{i}, \beta_{i}\right) \\
\mu_{i k} & \sim \mathcal{N}\left(v_{i}, s_{i}^{2}\right) \\
\sigma_{\varepsilon_{i}}^{2} & \sim \mathcal{I} \mathcal{G}\left(\alpha_{\varepsilon_{i}}, \beta_{\varepsilon_{i}}\right)
\end{aligned}
$$

with $k \in\{0,1\}$ representing the two classes of $X$ values and $i \in\{1,2, \ldots, m\}$ representing the $m$ data types.

(c) Clustering is performed using gene expression data by an unsupervised tight clustering algorithm [12] to obtain $z$ clusters.

2. For each iteration, sample $X$ from the posterior distribution:

$$
\begin{aligned}
\pi(X \mid \psi, \theta) & \propto p\left(\psi_{1}, \ldots, \psi_{m} \mid X, \theta_{1}, \ldots, \theta_{m}\right) p(X) \\
& =p(X) \prod_{i}^{m} p\left(\psi_{i} \mid X, \theta_{i}\right)
\end{aligned}
$$


which can be achieved according to Eq. (5) and Eq. (6), respectively. According to Eq. (11), for each element $x_{l}$ in $X, l=1,2, \ldots, e$, two probabilities can be computed: $p_{l}^{1}$ the probability that the element in $X$ is 1 and $p_{l}^{0}$ the probability that the element in $X$ is 0 . The probabilities are then normalized and compared with a number drawn from a uniform distribution to decide whether the new value takes 1 or 0 . This is to compute

$$
x_{l}=\left\{\begin{array}{c}
1, \quad \frac{p_{l}^{1}}{p_{l}^{1}+p_{l}^{0}}>=t \\
0, \quad \frac{p_{l}^{1}}{p_{l}^{1}+p_{l}^{0}}<t
\end{array}, \quad t \sim \operatorname{uniform}(0,1) .\right.
$$

3. Sample $\left\{\phi_{i} \mid i=1,2, \ldots, m\right\}$ from the posterior distribution:

The posterior distribution of $\phi$ is produced by the product of the likelihood function and the prior

$$
\begin{aligned}
\pi\left(\phi_{i} \mid \psi_{i}, X, \theta_{i}\right) & \propto p\left(\psi_{i} \mid \phi_{i}, X, \theta_{i}\right) p\left(\phi_{i} \mid X, \theta_{i}\right) \\
& =\mathcal{N}\left(\phi_{i}, \sigma_{\varepsilon_{i}}^{2}\right) \cdot \prod_{k=0,1} \mathcal{N}\left(\mu_{i k}, \sigma_{i k}^{2}\right) \\
& \propto \prod_{k=0,1} \mathcal{N}\left[\left(\frac{\psi_{i}}{\sigma_{\varepsilon_{i}}^{2}}+\frac{\mu_{i k}}{\sigma_{i k}^{2}}\right) \cdot\left(\frac{1}{\sigma_{\varepsilon_{i}}^{2}}+\frac{1}{\sigma_{i k}^{2}}\right)^{-1},\left(\frac{1}{\sigma_{\varepsilon_{i}}^{2}}+\frac{1}{\sigma_{i k}^{2}}\right)^{-1}\right]
\end{aligned}
$$

4. Sample $\left\{\theta_{i} \mid i=1,2, \ldots, m\right\}$ from posterior distributions:

$$
\begin{aligned}
\sigma_{i k}^{2} & \sim \mathcal{I} \mathcal{G}\left(\alpha_{i}+\frac{e_{k}}{2}, \beta_{i}+\frac{1}{2} \sum_{X=k}\left(\psi_{i}-\mu_{i k}\right)^{2}\right) \\
\mu_{i k} & \sim \mathcal{N}\left[\left(\frac{v_{i}}{s_{i}^{2}}+\frac{\sum_{X=k} \psi_{i}}{\sigma_{i k}^{2}}\right) \cdot\left(\frac{1}{s_{i}^{2}}+\frac{e_{k}}{\sigma_{i k}^{2}}\right)^{-1},\left(\frac{1}{s_{i}^{2}}+\frac{e_{k}}{\sigma_{i k}^{2}}\right)^{-1}\right] \\
\sigma_{\varepsilon_{i}}^{2} & \sim \mathcal{I} \mathcal{G}\left[\alpha_{\varepsilon_{i}}+\frac{e}{2}, \beta_{\varepsilon_{i}}+\frac{1}{2} \sum\left(\psi_{i}-\phi_{i}\right)^{2}\right]
\end{aligned}
$$

5. Iterate Step 2-4 until convergence.

Convergence is determined according to the Zellner and Min criteria [14]. In the case of Gibbs sampling the unknown parameters can be separated into two parts: $X$ and $\theta, \phi$. Therefore we have $\pi(X, \theta, \phi \mid \psi)=\pi(X \mid \theta, \phi, \psi) \pi(\theta, \phi \mid \psi)=\pi(\theta, \phi \mid X, \psi) \pi(X \mid \psi)$. Let iteration $b$ be the candidate stopping point of the chain, and $\pi_{b}(\hat{x} \mid \psi)$ be a smoothed empirical estimate, $\pi_{b}(\hat{X} \mid \psi)=$ $\sum_{j=1}^{b} \pi\left(X \mid \theta_{j}, \phi_{j}, \psi\right) / i$. The ratio of convergence

$$
\hat{\gamma}_{b}=\frac{\pi(X \mid \theta, \phi, \psi) \hat{\pi}_{b}(\theta, \phi \mid \psi)}{\pi(\theta, \phi \mid X, \psi) \hat{\pi}_{b}(X \mid \psi)}
$$

should be approximately one at convergence.

In summary, we empirically obtain the posterior distributions for the parameters and hyperparameters. If the Gibbs sampler has run sufficiently long, this algorithm produces a complete sample of the coefficients. The Gibbs sampler decomposes the whole set of parameters into three sets $\phi, \theta$ and $X$, since the form of random field we have chosen makes an exact sampling 
of $p\left(X \mid \phi_{1}, \ldots, \phi_{m}, \psi_{1}, \ldots, \psi_{m}\right)$ impossible. There are a few points we noted here for the proposed algorithm. First, the posterior of mean $\mu_{i k}$ depends on the data only through the sum of data $\sum \phi_{i}$, meaning that this data summary is sufficient from the data to estimate the unknown mean. Second, as the data size increases, the value of estimated mean will increasingly depend on the data and variance $\sigma_{i k}$, making the prior assumption less important. Last, it is possible that the set genes/proteins with regulatory roles are known for some genomes and therefore regulatory interactions only exist between them and all genes. This can greatly reduce the number of variables and speed up the algorithm,.

\section{Experiments}

Both synthetic data sets and biological data set are used for experimental evaluation. Biological data can only provide anecdotal evidence in network validation, since the knowledge of gene regulation is far from complete. It seems that we can use functional annotations as golden standard, but annotation information among different annotation databases is too inconsistent to support a large scale evaluation [15]. On the other hand, synthetic data sets can provide more controllable conditions to test an algorithm and a standard for benchmarking. However, to obtain meaningful results, the synthetic data need to share statistical characteristics with biological data.

For synthetic networks, gene expression data are generated for network reconstruction since they are the only synthetic data available. Based on these large-scale expression data sets, we compare the resultant networks from graphical Gaussian models (GGMs) [11] and the proposed algorithm. The synthetic expression data are generated by SynTReN [16], which is well suited for the purpose of testing network learning strategies [17]. By selecting subgraphs from previously described biological source networks, SynTReN produces synthetic gene expression data from a simulation of real regulatory network.

For real gene network, we integrate gene expression data, transcription factor binding data and protein-protein interaction data using the aforementioned framework for Yeast Saccharomyces Cerevisiae. Comparison of the resulting network and a golden standard network clearly shows the benefits of data integration.

\subsection{Synthetic networks}

The topologies of the synthetic networks are sub-sampled from Yeast transcriptional network [18] for the large networks, using a method named cluster addition (initial graph is selected as a randomly selected node and all of its neighbors). Combined with external conditions that trigger the network, the expression levels of genes in each experiment are generated according to the activities of their regulators.

Five synthetic networks are generated with Gaussian noise (noise level 0.15) and relatively large proportion of complex interaction (30\%). Details about the configuration of SynTReN are provided in supplementary Table 3 . Each with 25 samples/time points, the five synthetic networks consist of size 50 genes, 80 genes, 100 genes, 200 genes and 500 genes, respectively. An example of 200-gene synthetic network is plotted in Fig. 1(a). For each of the gene expression data set, we compare the resulting GGMs network with the result from the proposed 
BRFs model. ROC curves for altogether 10 tests are plotted in Fig. 1(b). The violet curves representing BRFs inference show superior performance to the green curves representing GGMs inference. For the synthetic data BRFs make use of its random field component but not the integration feature, since both of the methods take advantage of PCOR. In this way, the proposed BRFs model improves the resulting network, by imposing a cluster constraint in network inference.

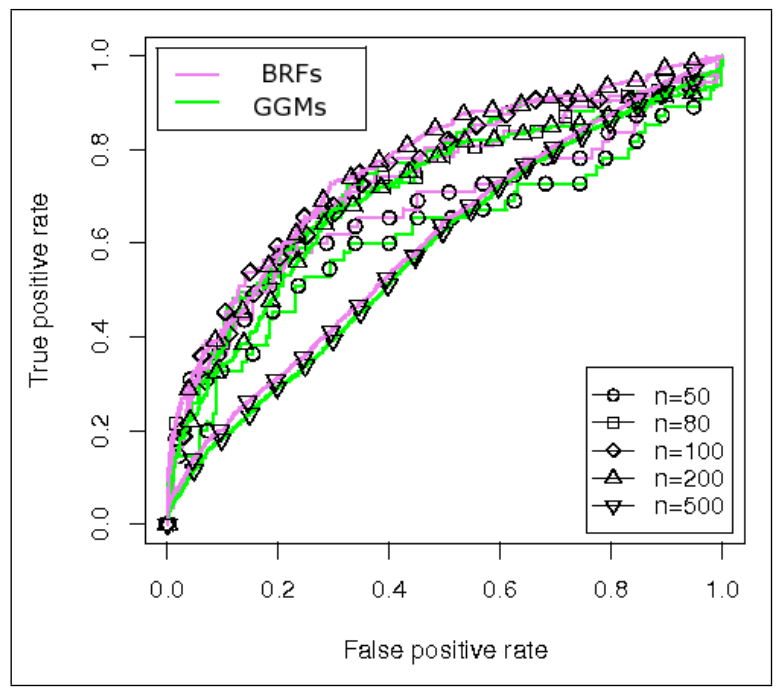

(a)

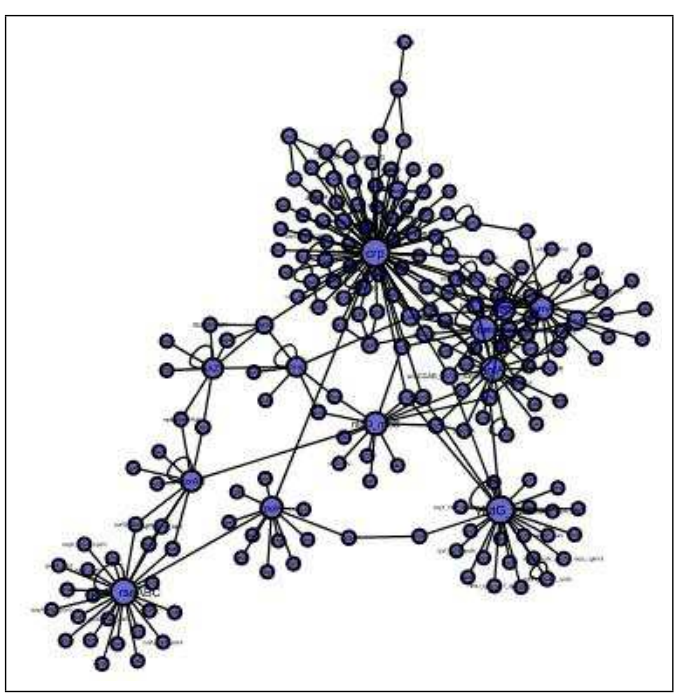

(b)

Figure 1: (a) ROC for comparison of GGMs and the proposed model on five synthetic networks of various size, (b) An example network of 200 genes

\subsection{Yeast Saccharomyces Cerevisiae regulatory network}

For the reconstruction of the Yeast regulatory network, three real data sets are integrated in this experiment. The result is compared to a golden standard network to evaluate the accuracy of the proposed method. The three data sets have their unique features: transcription factor binding data provide direct information to understand the regulators involved in transcription; protein-protein interaction (PPI) data reveal proteins that involved in the same pathway, as well as related to genomic level - interacting proteins are often co-expressed and co-localized to the same sub-cellular compartment. Both of these two data types are of certain degree of specificity and sparsity, but they can only describe the potential of interaction. In contrast, microarray expression time-series are a complementary source that provides dynamic information about the expressions of almost all genes under certain conditions in an organism. Although the data are known to be noisy, they reflect actual interactions in the biological process under analysis.

For gene expression data, the alpha factor arrest data set is selected from [19] since it has less missing data than the data set of the arrest of a cdc 15 temperature-sensitive mutant, yet longer time series than the data set of elutriation experiment. It consists of expression data of 6178 genes and 18 time points with $3.67 \%$ missing data. Although it is a relatively old data set, there are two reasons to use it. First, a number of works has used it in their evaluations because of its public availability, therefore providing a good comparison platform. Secondly, a good integration framework to combine heterogeneous data types is supposed to be able to amend for data set of poor quality. PPI data were downloaded from DIP database [20] containing 18,272 
interaction data from 4,985 Yeast proteins (as of Feb. 2008). PPI data stored in DIP were obtained through manual curation of the scientific literature including both direct and complex interactions. Transcription factor binding data consist of the binding of almost all known Yeast transcription factors monitored during cell growth in rich medium [21]. After excluding some probes for some computational reasons and problems with their microarry experiments, they provide binding data for 6229 genes across 203 transcription factors with $2.5 \%$ missing data.

For the golden standard network/ground truth, we selected a yeast regulatory network from a comprehensive study [22]. The network was assembled from literature and a large amount of data, then divided into condition specific sub-networks including cell cycle, sporulation, diauxic shift, DNA damage and stress response. Altogether it contains 7,074 regulatory relationships between 142 transcription factors and 3,420 target genes. In this paper the cell cycle subnetwork of 550 interactions among 296 genes is used as golden standard to compare with part of the resultant network.

To infer a cell-cycle specific network, we selected 909 genes by including the Spellman's 800 cell cycle's genes [19], Luscombe's 296 cell cycle's gene, and Price's 104 cell cycle genes [23]. Among these genes, there are 84 transcription factors. $2.7 \%$ of the gene expression data are missing for the 909 genes. There are 9 genes with $50 \%$ of their expression data missing and the corresponding data are discarded from use. The rest of missing data are imputed by weighted K-nearest neighbours (KNNimpute) shown to be robust for microarray data [24]. For this gene set, the available transcription binding data are transcription factors binding 902 genes. 782 protein-protein interactions are found among all genes, which again composes of only a small fraction of all possible interactions $(0.09 \%)$. All the data sets are available in the supplementary files.

BRFs inferred a network with 1,674 interactions between the 84 transcription factors and 669 genes, leaving 240 genes as irrelevant to the condition under study. The adjacency matrix is shown in Supplementary Fig. 3(a). Since the network is too large to visualize, we select a sub-network that contain 608 interactions among the same set of 296 genes as in the golden standard cell cycle network and plot it in Fig. 2(c). Given the golden standard network, we can now investigate on the power of data integration of the proposed method. For each data set, we assume there is a simple cut-off selection method and plot its corresponding ROC curves in Fig. 2(a). For example, since the binding data are the probabilities that a transcription factor binds to a gene, a cut-off threshold can be selected to include those interactions with higher probability than this threshold. Then the result of BRFs inference of $\left\{p_{l}^{1} \mid l=1,2, \ldots, e\right\}$, instead of binary matrix $X$, is plotted (red curve).

As it is shown in Fig. 2(a), the ROC curve (black) for gene expression PCOR is not very indicative of the true network, although comprehensive. This is consistent with previous finding that expression data are often too noisy and sensitive. For binding data, the violet curve implies relatively good quality, but because of the missing data (of the 70 transcription factors, only 57 present in the binding data), it cannot provide a good coverage. The PPI data is so sparse that only 194 PPI pairs were found among 296 genes, and therefore it constitutes only a small fraction of all possible interactions $(296 \times 296=87,616)$. The sparsity and poor quality of PPI data may be one of the reasons that it alone achieves low predictive performance. Another reason lies in that less direct relationships exist between PPI and the transcriptional network, since the PPI data can only indicate potentials for interactions rather than presence of interactions in the process under analysis. This is also consistent with previous findings [1]. Nonetheless, the 
inclusion of a data set of low relevance and high noise reflects the robustness of the proposed algorithm, since the resultant network is neither biased to noise nor affected by the irrelevant information.
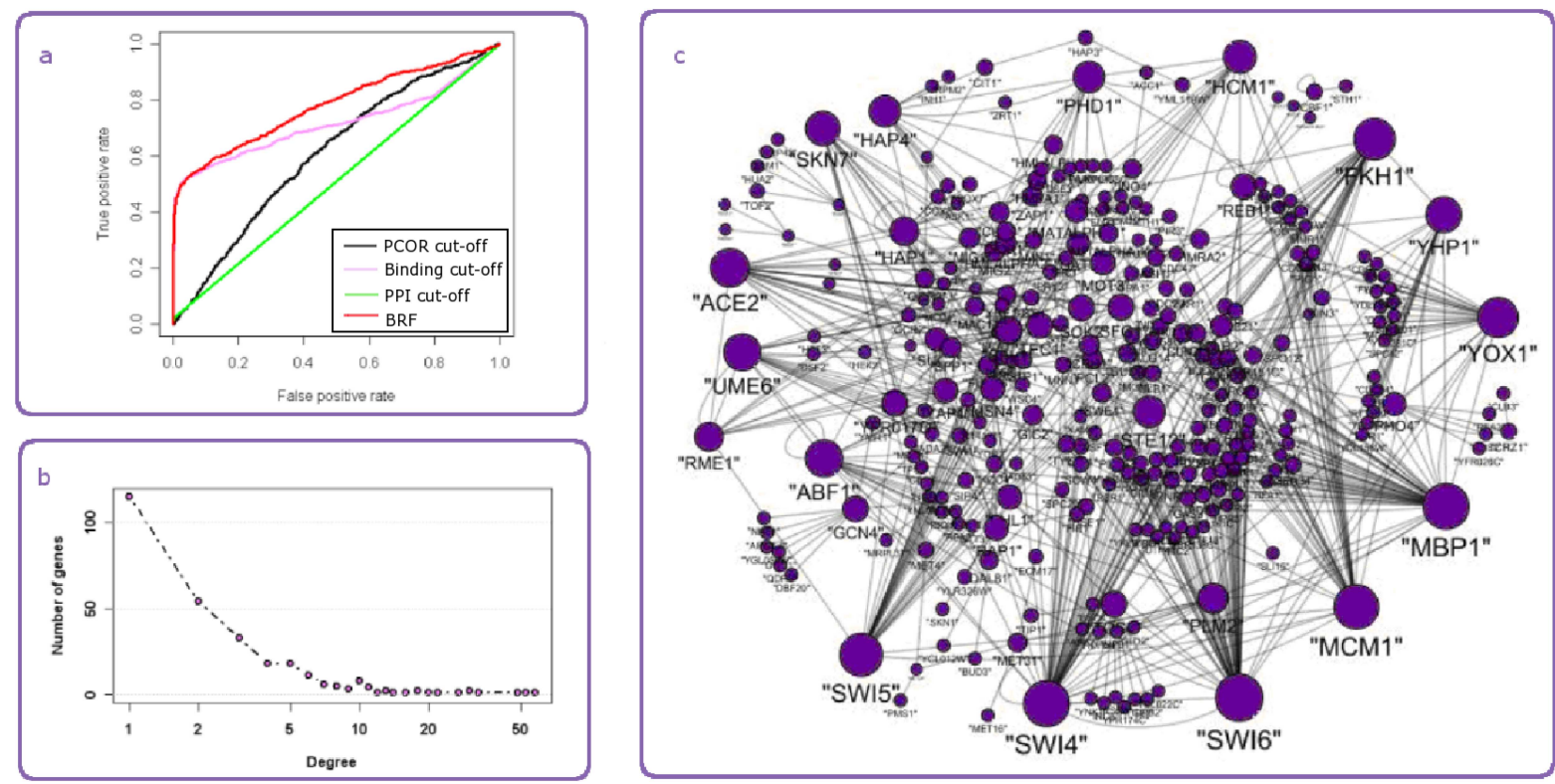

Figure 2: (a) ROC curves for threshold selection methods for the three data types used in network reconstruction and the resultant network by BRFs, (b) Connectivity degree distribution of the sub-network, $x$ axis shows the degree of connectivity of 296 nodes in log 2 scale, (c) The inferred cell cycle specific sub-network, size of the nodes indicate their degree of connectivity

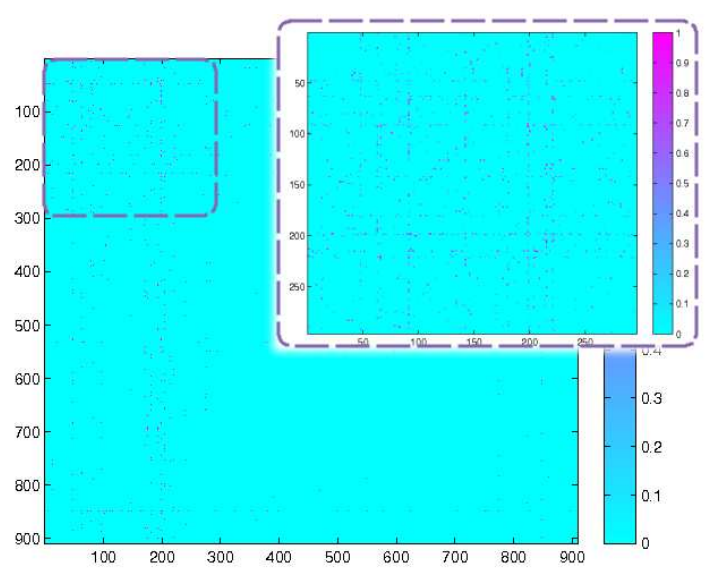

(a)

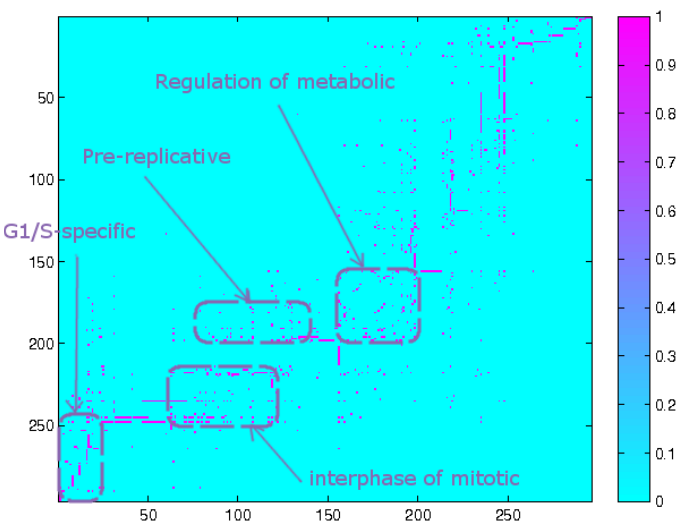

(b)

Figure 3: (a) The adjacency matrix of the inferred network, the part in dash frame corresponds to cell cycle specific sub-network, (b) The modularized adjacency matrix of the cell cycle specific sub-network

The distribution of connectivity degree of nodes in Fig. 2(b) shows a power-law tail. To look for the functional modules in such a sparse network, we study the clusters formed around hub-genes. The main hubs in this sub-network include "SWI4", "SWI6", "YOX1", "MCM1", "ACE2", etc. These 8 genes and their first neighbours cover $48 \%$ of 669 genes. We found these clusters significantly enriched with specific functions in gene ontology. The enrichment analysis results, plot of time series data of the eight transcription factors, and the genes they are 


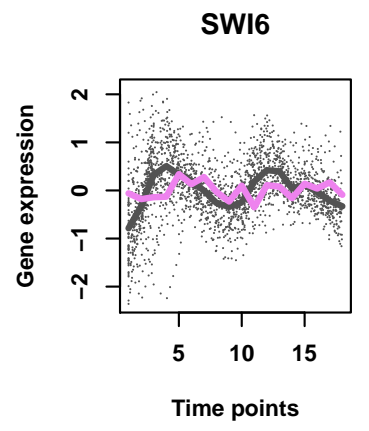

FKH1

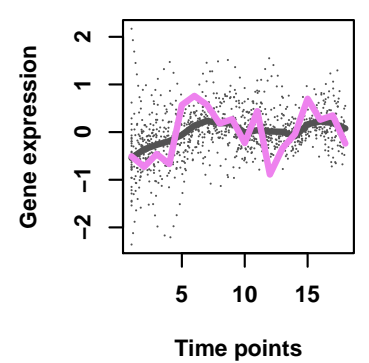

SWI4

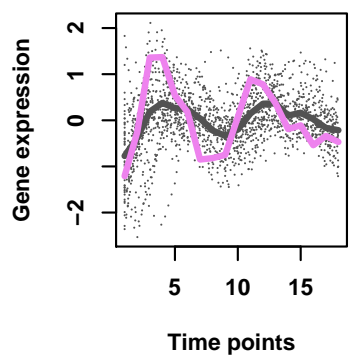

SWI5

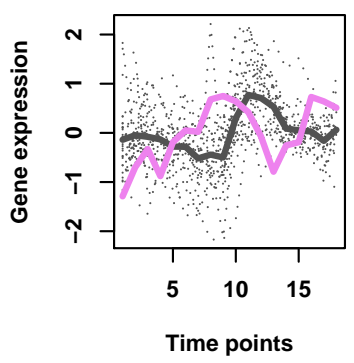

MBP1

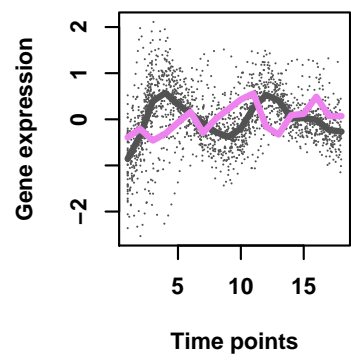

YOX1

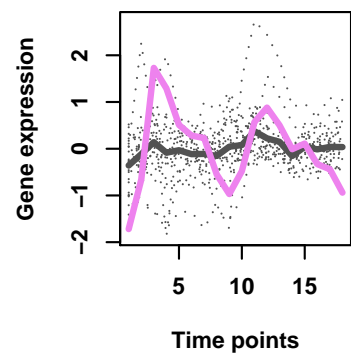

MCM1

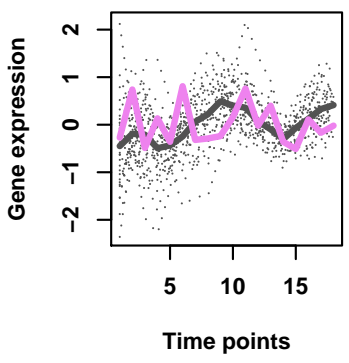

ACE2

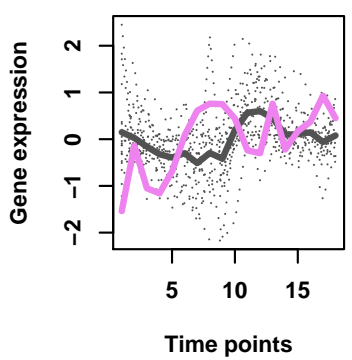

Figure 4: Plot of time series data of the eight transcription factors (pink) and the genes they are regulating (grey)

regulating are provided in Supplementary Table 1 and Supplementary Fig. 4. Also we analysis the adjacency matrix of the 296-gene sub-network. Finding modules in gene networks is nontrivial, since the degree of overlapping is high because of the existence of hubs. Four visible big clusters are found and illustrated in Supplementary Fig. 3(b). Analysis on the function of genes within these clusters reveals 4 phase-specific modules as shown in Table 2 in the supplement file.

\section{Conclusions}

Learning of large-scale regulatory networks is an important and challenging problem in bioinformatics. Genomic, transcriptomic and proteomic information are in massive production and they are related in one way or another. Although integrative analysis is promising in extracting deeper insights from diverse data sources, current methods are either bounded by the computational costs of microarray time series analysis, or the difficulties of adapting to new data sources.

The success of BRFs is a direct result of the inherent elegant yet straightforward integrative framework. Its flexibility enables unlimited heterogeneous data types to be integrated in a stochastic manner to facilitate robust estimation. Relative small number of parameters facilitates efficient estimation, even for a problem of large scale. As previously addressed, different data are of various formats and sparsity. BRFs propagates through modelling the two distributions in the available data sets without resorting to accounting for missing data, thus is more effective. In particular, the random fields component introduces a known feature of gene network for more accurate modelling. In summary, BRFs aims to achieve the limit of the available data, its power of integration is demonstrated through the experiments. 


\begin{tabular}{|c|c|c|c|c|}
\hline & TF.clusters & Ontology & P.values & bestGOs.goCounts \\
\hline 1 & SWI6 & G1/S-specific transcription in mitotic cell cycle & $6.68093393238038 \mathrm{e}-10$ & 12 \\
\hline 2 & SWI6 & regulation of cyclin-dependent protein kinase activity & $2.13810238799783 \mathrm{e}-09$ & 14 \\
\hline 3 & SWI6 & regulation of cell cycle & 4.02298103936134e-09 & 162 \\
\hline 4 & SWI6 & mitotic cell cycle & $3.53390486600709 \mathrm{e}-08$ & 231 \\
\hline 5 & SWI6 & regulation of kinase activity & $6.70586284584345 \mathrm{e}-08$ & 23 \\
\hline 6 & SWI4 & biological regulation & $2.40785075171311 \mathrm{e}-10$ & 783 \\
\hline 7 & SWI4 & G1/S-specific transcription in mitotic cell cycle & 3.37844099174296e-10 & 12 \\
\hline 8 & SWI4 & regulation of cellular process & $4.79119628041212 \mathrm{e}-10$ & 678 \\
\hline 9 & SWI4 & interphase of mitotic cell cycle & $5.49854796348663 \mathrm{e}-09$ & 92 \\
\hline 10 & SWI4 & regulation of cyclin-dependent protein kinase activity & $8.86641584383536 \mathrm{e}-08$ & 14 \\
\hline 11 & MBP1 & regulation of cyclin-dependent protein kinase activity & $7.46636474180021 \mathrm{e}-10$ & 14 \\
\hline 12 & MBP1 & regulation of kinase activity & $2.37003679824097 \mathrm{e}-08$ & 23 \\
\hline 13 & MBP1 & mitotic cell cycle & 3.23921949251363e-08 & 231 \\
\hline 14 & MBP1 & mitotic sister chromatid cohesion & $4.86909807679101 \mathrm{e}-07$ & 20 \\
\hline 15 & MBP1 & regulation of catalytic activity & $8.08691515638784 \mathrm{e}-07$ & 40 \\
\hline 16 & MCM1 & mitotic cell cycle & $3.36267031937684 \mathrm{e}-08$ & 244 \\
\hline 17 & MCM1 & biological regulation & $7.401233178589 \mathrm{e}-07$ & 783 \\
\hline 18 & MCM1 & interphase & $1.22247510142829 \mathrm{e}-06$ & 66 \\
\hline 19 & MCM1 & regulation of cell cycle & $1.82150388897989 \mathrm{e}-06$ & 162 \\
\hline 20 & MCM1 & pre-replicative complex formation & $4.17582220312772 \mathrm{e}-05$ & 13 \\
\hline 21 & FKH1 & cell cycle & $6.39276015731404 \mathrm{e}-07$ & 417 \\
\hline 22 & FKH1 & cell cycle phase & $2.06559855232857 \mathrm{e}-06$ & 302 \\
\hline 23 & FKH1 & interphase of mitotic cell cycle & 7.14048385347843e-06 & 92 \\
\hline 24 & FKH1 & chromosome segregation & $2.58642521052638 \mathrm{e}-05$ & 115 \\
\hline 25 & FKH1 & M phase of mitotic cell cycle & $4.34547001658344 \mathrm{e}-05$ & 126 \\
\hline 26 & SWI5 & $\begin{array}{l}\text { regulation of transcription from RNA polymerase II promoter by } \\
\text { carbon catabolites }\end{array}$ & $2.40342237875119 \mathrm{e}-05$ & 2 \\
\hline 27 & SWI5 & $\begin{array}{l}\text { negative regulation of transcription from RNA polymerase II promoter } \\
\text { by glucose }\end{array}$ & $2.40342237875119 \mathrm{e}-05$ & 2 \\
\hline 28 & SWI5 & negative regulation of transcription & $6.67551255482606 \mathrm{e}-05$ & 146 \\
\hline 29 & SWI5 & negative regulation of transcription from RNA polymerase II promoter & 0.000106197373740912 & 51 \\
\hline 30 & SWI5 & regulation of transcription, DNA-dependent & 0.000139377782444952 & 327 \\
\hline 31 & YOX1 & DNA replication & $9.48163239115115 \mathrm{e}-05$ & 112 \\
\hline 32 & YOX1 & mitotic cell cycle & 0.000464533646118637 & 244 \\
\hline 33 & YOX1 & cell cycle process & 0.00107472531481968 & 397 \\
\hline 34 & YOX1 & regulation of cellular process & 0.00137073530303063 & 678 \\
\hline 35 & YOX1 & mitosis & 0.00181625697370355 & 125 \\
\hline 36 & ACE2 & regulation of transcription from RNA polymerase II promoter & $9.58339994615866 \mathrm{e}-06$ & 206 \\
\hline 37 & ACE2 & regulation of biological process & $2.74054310418916 \mathrm{e}-05$ & 708 \\
\hline 38 & ACE2 & regulation of transcription & $3.51505697140104 \mathrm{e}-05$ & 350 \\
\hline 39 & ACE2 & negative regulation of transcription, DNA-dependent & 0.000185014832700214 & 141 \\
\hline 40 & ACE2 & regulation of cellular metabolic process & 0.000240908120079544 & 459 \\
\hline
\end{tabular}

Table 1: GO enrichment analysis results for the eight hubs and their neighbours.

\begin{tabular}{|c|c|c|c|c|c|}
\hline & Clusters & Ontology & P.values & bestGOs.intCounts & bestGOs.goCounts \\
\hline 1 & 1 & regulation of cellular metabolic process & $6.13691599053743 \mathrm{e}-12$ & 22 & 459 \\
\hline 2 & 1 & transcription, DNA-dependent & $1.03135849896327 \mathrm{e}-11$ & 22 & 471 \\
\hline 3 & 1 & regulation of transcription & $2.39583972690443 \mathrm{e}-10$ & 16 & 257 \\
\hline 4 & 1 & $\begin{array}{l}\text { regulation of transcription, mating-type } \\
\text { specific }\end{array}$ & $8.81634732337917 \mathrm{e}-10$ & 5 & 7 \\
\hline 5 & 1 & regulation of biological process & $5.73464467033518 \mathrm{e}-09$ & 21 & 589 \\
\hline 6 & 2 & $\begin{array}{l}\text { regulation of transcription, mating-type } \\
\text { specific }\end{array}$ & $2.17287437622652 \mathrm{e}-05$ & 3 & 7 \\
\hline 7 & 2 & transcription & $5.80455686891086 \mathrm{e}-05$ & 4 & 25 \\
\hline 8 & 2 & transcription, DNA-dependent & 0.000149160358659910 & 13 & 471 \\
\hline 9 & 2 & regulation of biological process & 0.000173131006726304 & 13 & 478 \\
\hline 10 & 2 & regulation of glycogen biosynthetic process & 0.00110693189084462 & 2 & 6 \\
\hline 11 & 3 & biological regulation & $9.67125173982757 \mathrm{e}-09$ & 34 & 783 \\
\hline 12 & 3 & $\begin{array}{l}\text { regulation of cyclin-dependent protein kinase } \\
\text { activity }\end{array}$ & $3.61718536321558 \mathrm{e}-08$ & 6 & 14 \\
\hline 13 & 3 & regulation of catalytic activity & $1.47009074750896 \mathrm{e}-07$ & 8 & 40 \\
\hline 14 & 3 & regulation of kinase activity & $1.08404764235319 \mathrm{e}-06$ & 6 & 23 \\
\hline 15 & 3 & interphase of mitotic cell cycle & $9.08365959742601 \mathrm{e}-05$ & 8 & 92 \\
\hline 16 & 4 & $\begin{array}{l}\text { regulation of nucleobase, nucleoside, } \\
\text { nucleotide and nucleic acid metabolic process }\end{array}$ & $5.30900948341711 \mathrm{e}-06$ & 17 & 396 \\
\hline 17 & 4 & regulation of metabolic process & $5.55172684599707 \mathrm{e}-06$ & 19 & 488 \\
\hline 18 & 4 & DNA replication & $9.17627854219876 \mathrm{e}-06$ & 9 & 112 \\
\hline 19 & 4 & G1/S-specific transcription in mitotic cell cycle & $1.05031913408718 \mathrm{e}-05$ & 4 & 12 \\
\hline 20 & 4 & transcription & $1.28742969964620 \mathrm{e}-05$ & 19 & 517 \\
\hline
\end{tabular}

Table 2: GO enrichment analysis results for the four clusters representing four phase-specific modules. 
This work is still ongoing. We provide here preliminary results from synthetic microarray data sets and Saccharomyces Cerevisiae data sets, and hope to extend the analysis to other organisms. Also, we are aware of the limitation of PCOR that in theory it is not as powerful as dynamic Bayesian network approaches when there are non-linear effects present in the data. However, given the paucity of samples available and the large scale of network, it is impossible for full order Bayesian inference. The performance of our network inference method using PCOR, as it is evaluated in the supplement file, is superior than 1st-order Bayesian network. Furthermore, we hope to refine the structure after relevant network modules are found in the subsequent analysis.

\section{Supplement: Microarry Time series Network Inference}

In data integration, gene expression time-series data are of special interest, not only because of their full coverage of the whole genome, but also because of their high dimensionality [25]. Such gene expression dynamics are important since they directly reveal the active components within the cell over time, thereby indicating certain interacting relationships. Regarding to how the time series data is used in network reconstruction, two types of methods, Dynamic Bayesian networks (DBNs) [1] and Graphical Gaussian models (GGMs) [11], account for a major part of current research.

DBNs have been widely used on time series expression data to provide information about system dynamics. A major concern about DBNs is its efficiency in terms of large-scale prediction on small-sample data. The type of model DBNs is based on, very often auto-regressive model, renders it a serious challenge when time series is short.

GMMs are undirected models well known to discriminate direct and indirect correlation between variables. In essence, partial correlation (PCOR) is used as the mathematical foundation for detecting meaningful interactions. GGMs have been applied for the reconstruction of gene networks by selecting significant coefficients of PCOR. Such coefficients correspond to direct interactions between involved genes. Schäfer and Strimmer [11] proposed an efficient estimation of PCOR and the use of False Discovery Rate (FDR) [26] for selecting cut-off for PCOR to form the network.

An efficient way of obtaining PCOR matrix, by the standard graphical theory, is through the inverse of covariance or correlation matrix [27]. However, classical time series analysis techniques are not readily applicable to transciptome data, in which the number of data points $n$ far exceeds the sample size $t$, since in this case the sample covariance and correlation matrices are not positively definite. Recently, an efficient way for computing PCOR was proposed by using only the $t-1$ eigenvectors corresponding to the $t-1$ non-zero eigenvalues of the covariance matrix [28]. Such a dimension reconstruction method is popular in signal processing community and known to be robust against noise.

GGMs have their own deficiency. An example of network inference by GGMs can be illustrated by plotting the distribution of PCOR in this case in Fig. 5. Although the true edges of the network does correspond to a group of coefficients that are significantly different from zero (pink), there are still many true negatives using FDR, since some of the PCOR coefficients with absolute values larger than the cut-off are not part of true map. Besides, the best selection of threshold still does not infer the $100 \%$ correct network because of the noise in the data (Fig. 5). 


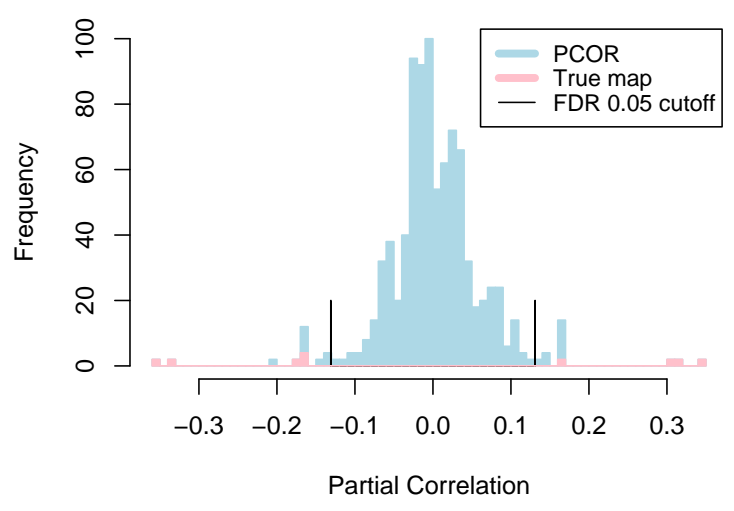

Figure 5: Distribution of all PCOR coefficients are plotted in blue histograms and pink histograms, which correspond to no edge and true edges in the true network. FDR is used in GGMs to determine PCOR coefficients that are significantly different from zero. A FDR cutoff of 5\% confidence is marked in the histogram (black line). The PCOR coefficient corresponding to true network edges (pink histogram) are not strictly distinguishable by such cut-off.

The definition of PCOR and the details for computing PCOR is provided in Section 2 and followed by a comparison of the performance of DBNs and GGMs in terms of gene network reconstruction in Section 3.

\subsection{Efficient computation of PCOR}

This section describes computation of partial correlation when the number of data points $n$ far exceed the sample size $t$, i.e. $n \gg t$.

In our framework, we propose to use partial correlation metric as the inference result from time series data. In the following section we will investigate into its accuracy and efficiency, in comparison to that of DBNs. By removing the linear effects from the rest of population, partial correlation can indicate whether a pair of variables directly interact with each other. Suppose $Y(t)=\left\{y_{1}(t), y_{2}(t), \ldots, y_{p}(t)\right\}$ is a multivariate stationary time series. The aim is to set up a graphical interaction model $G=(V, E)$ with the vertices $\{V\}$ as the components of the series and edges $\{E\}$ denoting pair-wise interactions. graph $G$ have such property:

$$
E(i, j) \subsetneq G \Leftrightarrow y_{i} \Perp y_{j} \mid Y_{-i j}
$$

Let $Y_{-i j}=\left\{y_{k} \mid k \neq i, j\right\}$, the linear effects of $Y_{-i j}$ is removed from $y_{i}$ by finding the parameter set $\theta_{i}=\left(\mu_{i}, \kappa_{i}\right)$ such that

$$
\hat{\theta}=\arg \min _{\theta} E\left\{y_{i}(t)-\mu_{i}-\sum_{u} \kappa_{i}(t-u) Y_{-i j}(u)\right\}^{2}
$$

The residuals of such regression is denoted as $\epsilon_{i}$. In the same way we define $\epsilon_{j}$. Thus the correlation between residuals $\epsilon_{i}$ and $\epsilon_{j}$ is the correlation between variables $y_{i}$ and $y_{j}$ conditioned on the others, i.e., partial correlation between $y_{i}$ and $y_{j}$. A direct interaction between $y_{i}$ and $y_{j}$ exists if and only if their partial correlation is significantly different from zero. When partial correlation is applied for network reconstruction, it provides solid mathematical foundation for finding meaningful interactions. It leads to the definition of such graph:

$$
E(i, j) \subsetneq G \Leftrightarrow \operatorname{cor}\left(\epsilon_{i}, \epsilon_{j}\right)=0
$$


An efficient way of obtaining partial correlation matrix, by the standard graphical theory, is through the inverse of covariance or correlation matrix [27]. Based on this theory, partial spectral coherence was proposed for frequency domain analysis of time series [27] and it can be obtained by the inverse of the spectral matrix [29]. However, these classical time series analysis techniques are not readily applicable to transciptome data, where the number of data points $n$ far exceed the sample size $t$, i.e. $n \gg t$. Since in this case the sample covariance and correlation matrices are not positively definite. Many efforts were spent on exploiting this field, either by restricting inference to a small number of genes [30], or limiting partial coefficient to limited order [31, 32], i.e., conditioned on only limited genes each time. Sampling technique such as bootstrapping is also proposed [11] in order to obtain point estimates of partial correlation coefficient. Recently, it was successfully proved that the partial correlation matrix maximizes the entropy of interaction system [28], and an efficient computation of partial correlation was proposed by using only the $t-1$ eigenvectors corresponding to the $t-1$ non-zero eigenvalues [28]. Such reconstruction method is popular in signal processing community and known to be robust against noise. Let

$$
V=\{v \in V, C v=\lambda v\}
$$

be the eigenvector of $C$, and $\lambda_{i}, i=1, \ldots, N$ be the eigenvalues. Since the spectral decomposition of covariance matrix $C$ is:

$$
C=M \Lambda M^{-1}, \quad\left\{\Lambda_{i i}\right\}=\lambda_{i}
$$

There are exactly $t-1$ non-zero eigenvalues, partial correlation matrix can be constructed in the non-zero eigenspace:

$$
P=C^{-1}=\left(M \Lambda M^{-1}\right)^{-1}=M \Lambda^{-1} M^{-1}
$$

$M$ is a matrix whose columns are made up of eigenvectors $v$, and $\Lambda$ is a diagonal matrix whose diagonal elements are the corresponding eigenvalues $\lambda$, therefore $\hat{P}$ can be reconstructed using the $t-1$ eigenvectors corresponding to $\left\{\lambda_{1}, \lambda_{2}, \ldots, \lambda_{t-1}\right\}$.

\subsection{Simulation set up}

Evaluation was conducted under two R packages: GeneNet [11] and G1DBN [33]. GeneNet imputes graphical Gaussian models (GGMs) by choosing significant partial correlations, while G1DBN use Dynamic Bayesian Networks inference (DBNs) on gene expression data with 1st order condition dependencies.

Auto-regressive data. First-order autoregressive model is used to generate time series data:

$$
X_{t}=A X_{t-1}+B+\epsilon_{t}
$$

where $\epsilon_{t}$ has a multivariate Gaussian distribution $N_{t}(0, \Sigma)$. Constant matrix $\mathrm{B}$, elements of diagonal matrix $\Sigma$, and $\eta e$ randomly chosen elements of transition matrix A, are uniformly drawn from a pre-fixed range of values, that is,

$$
A_{\eta e} \sim U\left(\left[-a_{\max },-a_{\min }\right],\left[a_{\min }, a_{\max }\right]\right),
$$

$B \sim U\left(b_{\min }, b_{\max }\right), \Sigma_{\text {diag }} \sim U\left(\sigma_{\min }, \sigma_{\max }\right)$, respectively. Then the time series data are generated according to Eq. (ar1). For cross-validation five data sets of 50 nodes and 25 time points were obtained with varied $\sigma_{\min }, \sigma_{\max }, b_{\min }, b_{\max }$ and $\eta=0.01 \sim 0.05$. 
Gaussian data. This method first set up a simulated partial correlation matrix $P$, by drawing coefficients from $U(-1,1)$ for $\eta e$ randomly chosen elements of $P$, in which process the topology of network is also constructed. To ensure $P$ is positive-definite, the diagonal elements are computed as follows:

$$
p_{i i}=\sum_{j, j \neq i} p_{i j}+\epsilon
$$

with $\epsilon$ is a small constant. $P$ can then be inverted to obtain covariance matrix $\Sigma=P^{(-1)}$ which is also positive-definite. Upon obtaining $C$, time series data can be generated following the multivariate normal distribution $N(0, \Sigma), X=A Q^{T}, A$ being the Cholesky decomposition of $\Sigma$ such that $A A^{T}=\Sigma$, and $Q$ a vector of independent standard normal variables of length $T$. For cross-validation five data sets of 50 nodes and 25 time points were obtained with varied $\eta=0.01 \sim 0.05$.

SynTReN. We varied some of the parameters of SynTReN and others set to default. Level of added noise and proportion of complex interactions are set to increase monotonically, resulting in an increasing level of complexity. The parameter settings are provided in Table 3, supplement. Instead of using the normalized data generated by SynTReN, we make use of the quantile normalization of the $\log _{2}$ ratio of raw data.

\begin{tabular}{|l|l|l|l|l|l|}
\hline Dataset & 1 & 2 & 3 & 4 & 5 \\
\hline Background Nodes & 50 & 50 & 50 & 50 & 50 \\
\hline Bio Noise & 0.02 & 0.05 & 0.08 & 0.1 & 0.1 \\
\hline Exp Noise & 0.02 & 0.05 & 0.08 & 0.1 & 0.1 \\
\hline Noise on correlated input & 0.02 & 0.05 & 0.08 & 0.1 & 0.1 \\
\hline Fraction of complex interactions & 0.1 & 0.1 & 0.2 & 0.2 & 0.3 \\
\hline
\end{tabular}

Table 3: Simulated data by SynTReN. Five datasets of $n=100$ nodes and 25 timepoints are generated. Parameter settings (set as default except the following

\subsection{Experiments on synthetic data}

For auto-regressive simulator and GGMs simulator each of the network consists of 50 genes and 25 time points. For SynTReN, each of the networks consists of 100 genes and 25 measurements/time points.

To evaluate the prediction power of DBNs and GGMs, we plot ROC curves [34, 35] in Fig. 6. The area under the ROC curve, AUC, represents the accuracy of the prediction. Although such comparison was reported before [36, 37], an important fact was neglected, that gene expression time series data are far shorter from other biological time series data such as neuron data. For example, DBNs implementation is usually designed for hundreds or thousands of samples, e.g., the 2000 sample points of simulated data in [36, 38]. Limitation of microarray experimental costs prohibits such technique from exploring gene expression data. Moreover, the computation of DBNs would be too heavy for more than a hundred variables. Since the simulated data are generated following the characteristic assumption of small sample, we hope to cast light on this particular aspect.

From the ROC curves in Fig. 6, we found that each of the techniques is well adapted its default type of data: DBNs is sensitive to data generated from auto-regressive model, while GGMs 
shows excellent performance on Gaussian data. Obviously, when the data satisfy the assumption of the models, we can expect the corresponding technique perform well. Therefore, an important question pertains to which assumption best describes gene expression data. If the simulated data by SynTReN best approximate the nature of microarry time series, GGMs offers better prediction than first-order DBNs, as it can be seen from Fig. 6(f). In this respect, it is promising to use PCOR to replace gene expression data in our integration framework because of its efficiency and prediction power.

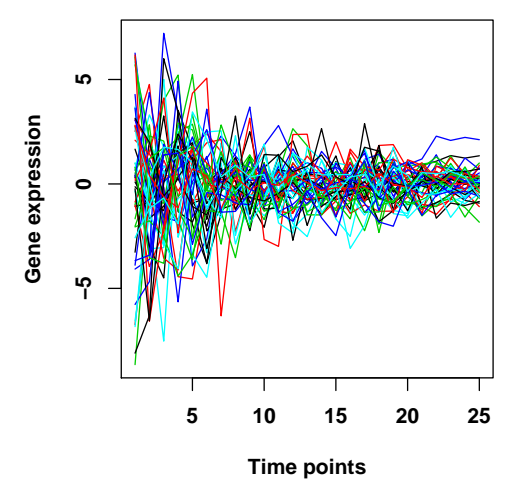

(a) Auto-regressive data

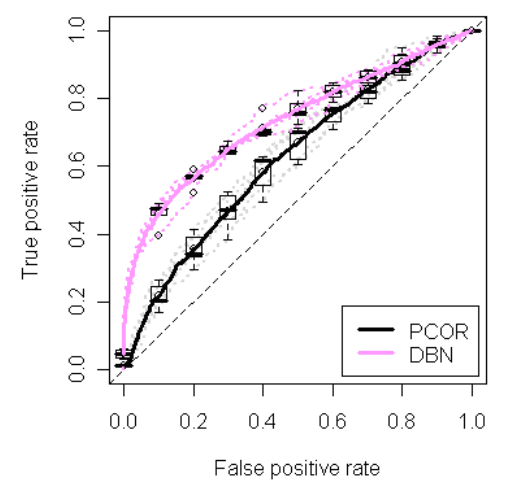

(d) ROC curves

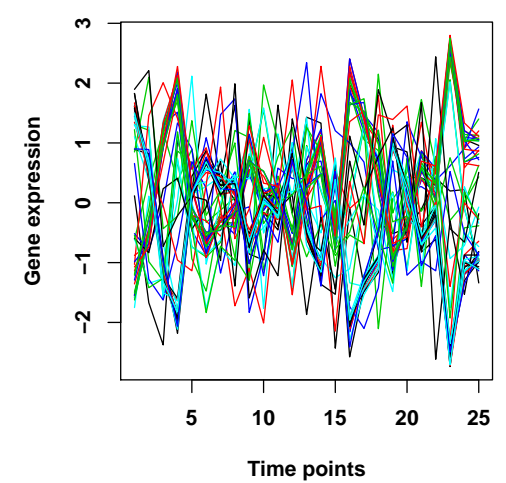

(b) Gaussian simulated data

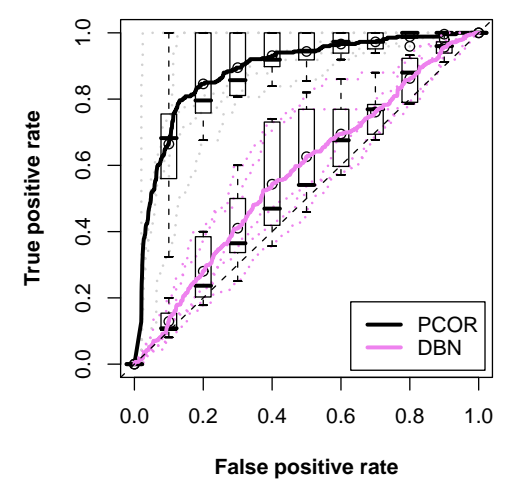

(e) ROC curves

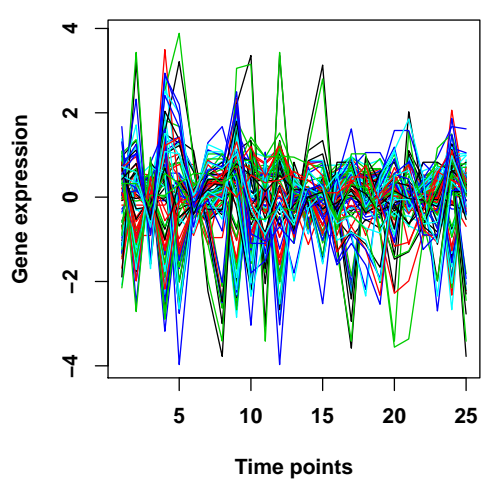

(c) SynTReN data

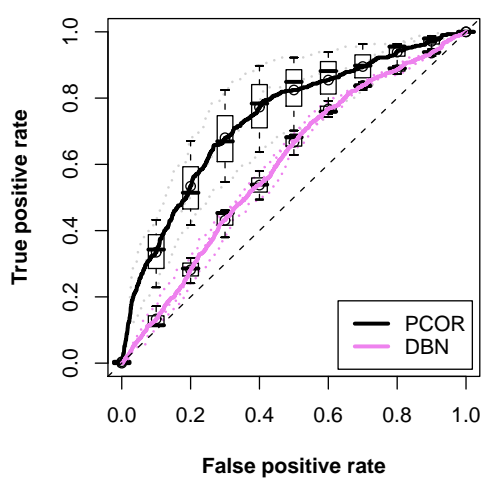

(f) ROC curves

Figure 6: Comparisons of performance of GGMs/PCOR and DBNs in the analysis of synthetic networks.

\section{References}

[1] Allister Bernard and Alexander J. Hartemink. Informative structure priors: joint learning of dynamic regulatory networks from multiple types of data. Pacific Symposium on Biocomputing, pages 459-70, 2005.

[2] Albert Tarantola. Popper, Bayes and the inverse problem. Nature Physics, 2(8):492-494, 2006.

[3] N. Nariai, S. Kim, S. Imoto, and S. Miyano. Using protein-protein interactions for refining gene networks estimated from microarray data by Bayesian networks. Pacific Symposium on Biocomputing, pages 336-47, 2004. 
[4] E. Segal, M. Shapira, A. Regev, D. Pe'er, D. Botstein, D. Koller, and N. Friedman. Module networks: identifying regulatory modules and their condition-specific regulators from gene expression data. Nature Genetics, 34(2):166-176, 62003.

[5] Ning Sun, Raymond J. Carroll, and Hongyu Zhao. Bayesian error analysis model for reconstructing transcriptional regulatory networks. Proceedings of the National Academy of Sciences, 103(21):7988-7993, 2006.

[6] Eric H. Davidson, Jonathan P. Rast, Paola Oliveri, Andrew Ransick, Cristina Calestani, Chiou-Hwa Yuh, Takuya Minokawa, Gabriele Amore, Veronica Hinman, Cesar ArenasMena, Ochan Otim, C. Titus Brown, Carolina B. Livi, Pei Yun Lee, Roger Revilla, Alistair G. Rust, Zheng Jun Pan, Maria J. Schilstra, Peter J. C. Clarke, Maria I. Arnone, Lee Rowen, R. Andrew Cameron, David R. McClay, Leroy Hood, and Hamid Bolouri. A genomic regulatory network for development. Science, 295(5560):1669-1678, 2002.

[7] O. G. Troyanskaya, K. Dolinski, A. B. Owen, R. B. Altman, and D. Botstein. A Bayesian framework for combining heterogeneous data sources for gene function prediction (in Saccharomyces cerevisiae). Proceedings of the National Academy of Sciences, 100(14):8348-8353, 2003.

[8] Daniel R. Rhodes, Scott A. Tomlins, Sooryanarayana Varambally, Vasudeva Mahavisno, Terrence Barrette, Shanker Kalyana-Sundaram, Debashis Ghosh, Akhilesh Pandey, and Arul M. Chinnaiyan. Probabilistic model of the human protein-protein interaction network. Nature Biotechnology, 23(8):951-959, 2005.

[9] Hawoong Jeong, Sean P. Mason, Albert-Laszlo Barabasi, and Zoltan N. Oltvai. Lethality and centrality in protein networks. Nature, 411:41-42, 2001.

[10] M. Ashburner, C. A. Ball, J. A. Blake, D. Botstein, H. Butler, J. M. Cherry, A. P. Davis, K. Dolinski, S. S. Dwight, J. T. Eppig, M. A. Harris, D. P. Hill, L. Issel-Tarver, A. Kasarskis, S. Lewis, J. C. Matese, J. E. Richardson, M. Ringwald, G. M. Rubin, and G. Sherlock. Gene ontology: tool for the unification of biology. The Gene Ontology Consortium. Nature Genetics, 25:25-29, 2000.

[11] Juliane Schäfer and Korbinian Strimmer. An empirical Bayes approach to inferring largescale gene association networks. Bioinformatics, 21(6):754-764, 32005.

[12] Yinyin Yuan and Chang-Tsun Li. Partial mixture model for tight clustering of gene expression time-course. BMC Bioinformatics, 9:287, 2008.

[13] Jeff Gill. Bayesian methods: a social and behavioral sciences approach. Chapman \& Hall/CRC, Boca Raton, FL, USA, 2002.

[14] Arnold Zellner and Chung-Ki Min. Gibbs sampler convergence criteria. Journal of the American Statistical Association, 90(431):921-927, 91995.

[15] Chad Myers, Daniel Barrett, Matthew Hibbs, Curtis Huttenhower, and Olga Troyanskaya. Finding function: evaluation methods for functional genomic data. BMC Genomics, 7(1):187, 2006. 
[16] Tim van den Bulcke, Koenraad van Leemput, Bart Naudts, Piet van Remortel, Hongwu Ma, Alain Verschoren, Bart de Moor, and Kathleen Marchal. SynTReN: a generator of synthetic gene expression data for design and analysis of structure learning algorithms. BMC Bioinformatics, 7(1):43, 12006.

[17] Tom Michoel, Steven Maere, Eric Bonnet, Anagha Joshi, Yvan Saeys, Tim van den Bulcke, Koenraad van Leemput, Piet van Remortel, Martin Kuiper, Kathleen Marchal, and Yves van de Peer. Validating module network learning algorithms using simulated data. BMC Bioinformatics, 8(Suppl 2):S5, 2007.

[18] Nabil Guelzim, Samuele Bottani, Paul Bourgine, and Francois Képès. Topological and causal structure of the yeast transcriptional regulatory network. Nature Genetics, 31(1):60-63, 42002.

[19] P. T. Spellman, G. Sherlock, M. Q. Zhang, V. R. Iyer, K. Anders, M. B. Eisen, P. O. Brown, D. Botstein, and B. Futcher. Comprehensive identification of cell cycle-regulated genes of the yeast Saccharomyces cerevisiae by microarray hybridization. Molecular Biology of the Cell, 9(12):3273-3297, 1998.

[20] L. Salwinski, C. S. Miller, A. J. Smith, F. K. Pettit, J. U. Bowie, and D. Eisenberg. The Database of Interacting Proteins: 2004 update. Nucleic Acids Research, 32:449-451, 2004.

[21] Christopher T. Harbison, D. Benjamin Gordon, Tong Ihn Lee, Nicola J. Rinaldi, Kenzie D. Macisaac, Timothy W. Danford, Nancy M. Hannett, Jean-Bosco Tagne, David B. Reynolds, Jane Yoo, Ezra G. Jennings, Julia Zeitlinger, Dmitry K. Pokholok, Manolis Kellis, P. Alex Rolfe, Ken T. Takusagawa, Eric S. Lander, David K. Gifford, Ernest Fraenkel, and Richard A. Young. Transcriptional regulatory code of a eukaryotic genome. Nature, 431:99-104, 92004.

[22] Nicholas M. Luscombe, M. Madan Babu, Haiyuan Yu, Michael Snyder, Sarah A. Teichmann, and Mark Gerstein. Genomic analysis of regulatory network dynamics reveals large topological changes. Nature, 431:308-12, 2004.

[23] Clive Price, Kim Nasmyth, and Tillman Schuster. A general approach to the isolation of cell cycle-regulated genes in the budding yeast, Saccharomyces cerevisiae. Journal of Molecular Biology, 218(3):543-556, 1991. Cited By (since 1996): 33.

[24] Olga G. Troyanskaya, Michael Cantor, Gavin Sherlock, Patrick O. Brown, Trevor Hastie, Robert Tibshirani, David Botstein, and Russ B. Altman. Missing value estimation methods for DNA microarrays. Bioinformatics, 17(6):520-525, 62001.

[25] Robert Clarke, Habtom W. Ressom, Antai Wang, Jianhua Xuan, Minetta C. Liu, Edmund A. Gehan, and Yue Wang. The properties of high-dimensional data spaces: implications for exploring gene and protein expression data. Nature Reviews Cancer, 8(1):37-49, 12008.

[26] Yoav Benjamini and Yosef Hochberg. Controlling the false discovery rate: A practical and powerful approach to multiple testing. Journal of the Royal Statistical Society. Series B (Methodological), 57(1):289-300, 1995. 
[27] David R. Billinger. Time series: data analysis and theory. International series in decision processes. Holt, Rinehart and Winston, New York, NY, USA, 1981.

[28] Timothy R. Lezon, Jayanth R. Banavar, Marek Cieplak, Amos Maritan, and Nina V. Fedoroff. Using the principle of entropy maximization to infer genetic interaction networks from gene expression patterns. Proceedings of the National Academy of Sciences, 103(50):19033-19038, 112006.

[29] R. Dahlhaus. Graphical interaction models for multivariate time series. Metrika, 51:157$172,1999$.

[30] Junbai Wang, Ola Myklebost, and Eivind Hovig. Mgraph: graphical models for microarray data analysis. Bioinformatics, 19(17):2210-2211, 2003.

[31] Anja Wille, Philip Zimmermann, Eva Vranová, Andreas Fürholz, Oliver Laule, Stefan Bleuler, Lars Hennig, Amela Prelic, Peter von Rohr, Lothar Thiele, Eckart Zitzler, Wilhelm Gruissem, and Peter Bühlmann. Sparse graphical Gaussian modeling of the isoprenoid gene network in Arabidopsis thaliana. Genome Biology, 5(11):R92, 2004.

[32] Alberto de la Fuente, Nan Bing, Ina Hoeschele, and Pedro Mendes. Discovery of meaningful associations in genomic data using partial correlation coefficients. Bioinformatics, 20(18):3565-3574, 2004.

[33] Sophie Lebre. Inferring dynamic genetic networks with low order independencies, 2007. http://www . citebase .org/abstract?id=oai : arXiv .org:0704 . 2551.

[34] James P. Egan. Signal detection theory and ROC analysis. Academic Press Series in Cognition and Perception. Academic Press, New York, NY, USA, 1975.

[35] Tom Fawcett. An introduction to roc analysis. Pattern Recognition Letters, 27(8):861$874,2006$.

[36] Alexander J. Hartemink. Reverse engineering gene regulatory networks. Nature Biotechnology, 23(5):554-5, 52005.

[37] Katia Basso, Adam A. Margolin, Gustavo Stolovitzky, Ulf Klein, Riccardo Dalla-Favera, and Andrea Califano. Reverse engineering of regulatory networks in human B cells. Nature Genetics, 37:382-390, 32005.

[38] Jing Yu, V. Anne Smith, Paul P. Wang, Alexander J. Hartemink, and Erich D. Jarvis. Advances to Bayesian network inference for generating causal networks from observational biological data. Bioinformatics, 20(18):3594-3603, 72004. 